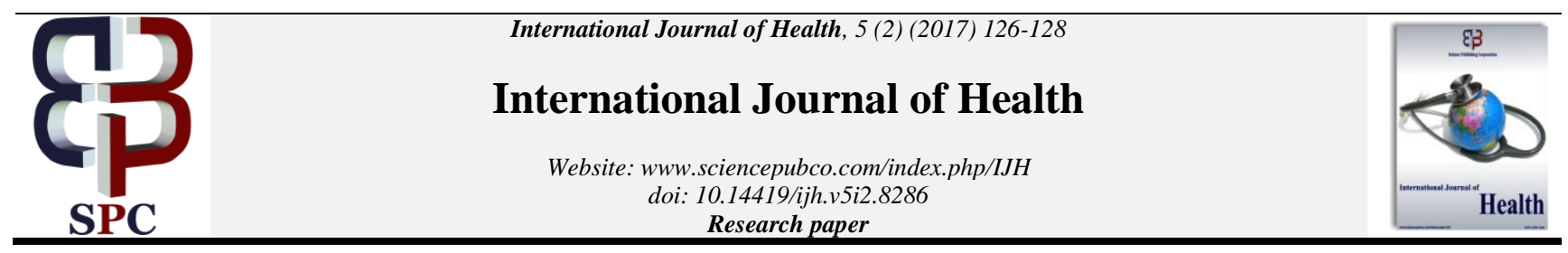

\title{
Implementation and evaluation of communication guidelines for the nurses caring for patients diagnosed with tuberculosis
}

\author{
Esther Kamenye $^{1 *}$, Scholastika lipinge ${ }^{2}$, Agnes Van Dyk ${ }^{3}$ \\ ${ }^{1}$ Department of Community Health, School of Nursing, University of Namibia (UNAM) Windhoek, Namibia \\ ${ }^{2}$ Clara Barton School of Nursing, Welwitchia University(WU), Windhoek, Namibia \\ ${ }^{3}$ Department of General Nursing Science, International University of Management (IUM), Windhoek, Namibia \\ *Corresponding author E-mail: kamenyene@gmail.com
}

\begin{abstract}
This article focuses on the process of the implementation and evaluation of the guidelines on communication to enhance the communication skills of the nurses who are caring for the patients diagnosed with tuberculosis. This article consists of two sections. The first section deals with the implementation of the communication guidelines and the second section evaluates the implementation and the outcomes of the evaluation of the guidelines. The guideline's document was completed during a two-day training workshop held at the Katutura Health Centre in Namibia.
\end{abstract}

Keywords: Communication Skills; Evaluation; Guidelines; Implementation; Nurses.

\section{Introduction}

Tuberculosis (TB) has been a significantly major public health threat worldwide. Two billion people are infected with Mycobacterium tuberculosis, nine million new cases are reported every year, and two million die every year from the disease (World Health Organization [WHO], 2003). The incidence of tuberculosis has increased since the mid-1980s and continues to grow by one per cent each year, despite the fact that tuberculosis is a preventable, treatable, and curable disease (WHO, 2006). In 2004, Namibia reported a tuberculosis case notification rate of 822 persons per 100000 population, and it was the highest incidence ever reported in the world (Ministry of Health and Social Services [MoHSS], 2006, p.1).

The Government of the Republic of Namibia has pursued vigorous TB programmes, which aim at eliminating TB. Commitment in this regard has been shown by the following efforts: purchasing of all anti-TB medicine, paying for all sputum examinations, and providing the infrastructure and human resources for TB treatment. International and non-governmental organisations are also supporting the efforts of the government. Despite all these strategies, problems are still existing, for instance, the tuberculosis case notification rate in Namibia has steadily increased from 656 per 100000 in 1997 to 722 per 100000 in 2007 (MoHSS, 2007/2008, p. 9). Another problem arises is the emergence of drug-resistant TB of 268, which has been reported in Namibia by the end of 2007 (MoHSS, 2007/2008, p. 16). This statistical information indicates that there are problems in the management of tuberculosis in the country, particularly in the Khomas Region.

According to Clark (2008), many problems that occur in any organization in respect of management are the direct results of people who are failing to communicate properly. Ineffective communication causes problems, and leads to misunderstanding, confusion, and failure of excellent plans. Therefore, good communica- tion behavior of the nurses who are caring for patients with tuberculosis are very important to the management of tuberculosis. In Namibia, there are no guidelines on communication to enhance the communication skills of the nurses who are caring for patients diagnosed with tuberculosis. The National Guidelines for the Management of Tuberculosis emphasises the important aspects that need to be provided to each patient diagnosed with tuberculosis on every visit (MoHSS, 2006). However, it is the researcher's view that even though the nurses know what substantive messages to convey to their patients, they may not possess good communication skills to exactly communicate TB health information most effectively, especially when there are no guidelines on communication implemented for reference purposes.

Moreover, the nurses caring for patients diagnosed with tuberculosis have different training backgrounds; some are registered nurses, while some of them are enrolled nurses (MoHSS, 2009/2010). It may lead to different levels of communication, especially when there are no standardised communication guidelines to enhance their communication skills to the same level.

Communication guidelines for nurses who are caring for patients diagnosed with tuberculosis can be effective to enhance their communication skills. Therefore, a study was conducted mainly to develop the guidelines.

\section{Methodology}

This article is extracted from the author's study, "Guidelines to enhance the communication skills of nurses caring for the patients diagnosed with tuberculosisat public health facilities in the Khomas region of Namibia.To address the purpose of the study, a research project was conducted, which was quantitative, exploratory, and contextual in nature. The study was conducted into four phases, starting with an exploration and description of communication process between nurses and patients diagnosed with tuberculosis in Phase 1, followed by the development of a conceptual 
framework for effective communication in phase two. Phase 3 involved the process of developing guidelines, and Phase 4 focused on the implementation and evaluation of these guidelines. The population and the sample of the study were the same (thirty nurses and thirty patients diagnosed with TB from all the public health facilities in the Khomas Region). The data collection method employed was observation using a checklist, followed by individual semi-structured face-to-face interviews with nurses, and also with the patients diagnosed with tuberculosis. The quality of research and research instrument were determined by its validity and reliability. Data obtained were analysed by using the EpiInfo $^{\mathrm{TM}}$ software package, and content analysis.

The main findings established that nurses who were caring for patients diagnosed with tuberculosis exhibited inadequate communication skills in the following areas: creating a conducive environment for communication, assessing and understanding the patients' mood and level of understanding, listening, questioning, constructive feedback, understanding of non-verbal communication, respect, and empathy for patients. The study results also revealed that patients diagnosed with tuberculosis had inadequate knowledge about TB. The majority of patients diagnosed with TB did not know the type of TB they had, and they perceived alcohol and smoking as the causes of TB. From the findings, the researcher developed guidelines for communication that were reviewed and validated by the guidelines development group.

This article is only focused on Phase 4 of the study which was the implementation and evaluation of the draft guidelines on communication.

\section{Objective}

To implement and evaluate the draft guidelines for communication of the nurses caring for patients diagnosed with tuberculosis in public health facilities in the Khomas Region of Namibia.

\section{Guidelines on communication}

The guidelines on communication that were implemented and evaluated were focused on the following areas:

- Creating a conducive atmosphere for communication;

- Assessing and understanding the patients' mood and TB situation;

- Listening skills;

- Questioning skills;

- Constructive feedback skills;

- Understanding non-verbal communication skills; and

- Expressing respect and empathy to patients.

\section{Implementation}

The implementation consisted of a work plan that was drawn up to provide guidelines about the content to be implemented; as well as when, by whom, and how it should be implemented (Watson, 2011). Effective planning and preparation create a conducive environment that facilitates active participation from the people (Rew, 2005). In this study, effective preparation for the implementation of the draft guidelines was employed whereby the researcher conducted a two-day training workshop at the Katutura Health Centre Conference Hall in Windhoek for the nurses who were caring for patients with tuberculosis at the public health facilities in the Khomas Region. The rationale of the workshop was to train nurses who were caring for patients with tuberculosis on how to execute the draft guidelines for communication daily at any TB department in the Khomas Region. Seven nurses from seven public health facilities in the Khomas Region of Namibia were trained. The content of the training was mainly focused on the following basic communication skills: creating a conducive atmosphere for communication; assessing and understanding the patients' mood, listening; questioning; understanding non-verbal communication skills and on how to express respect and empathy to the patients. Name of the health facilities and the number of nurses trained at each health facility were as follow:

\section{Katutura Health Centre 01}

Khomasdal Clinic 01

Wanaheda Clinic 01

Hakahana Clinic 01

Okuryangava Clinic 01

Robert Mugabe Clinic 01

Donkerhoek Clinic 01

The trained nurses were allowed three months to implement the content of the draft guidelines for communication during their daily care of each patient diagnosed with tuberculosis and also of the patients' DOT supporters. During those months, the researcher monitored the implementation by conducting monthly support visits and by providing in-service training to the same nurses. The visits also afforded them an opportunity to seek clarification about the draft guidelines for communication from the researcher. Furthermore, the researcher availed herself by providing the nurses with her contact number to contact her whenever they experienced any problem in relation to either communication or tuberculosis management.

\section{Evaluation}

Three months after the implementation of the draft guidelines for communication, the researcher conducted an evaluation process.According to Pretorius (2008), evaluation is the assessment of whether the activities have been congruent to the set programme In this study, the main aim of the evaluation was to establish whether the communication skills of the nurses who were caring for the patients diagnosed with tuberculosis improved after training. Furthermore, evaluation was also done to assess whether the TB knowledge about the patients diagnosed with tuberculosis had improved after receiving care from the trained nurses. In short, evaluation was conducted to establish whether the developed guidelines for communication indeed brought some change to the TB program.

To accomplish that, the researcher collected data by an observational method by using the same tool (checklist and interview with semi-structural open-ended questions) she had used during the situational analysis during the first phase of this study.

\section{The results of the evaluation}

It was evident from the evaluation findings that the draft guidelines for communication had brought about a significant improvement in the communication skills of the nurses who were caring for the patients with tuberculosis at the public health facilities in the Khomas Region of Namibia.

From the evaluation findings, one could conclude that the following communication skills of the nurses enhanced after they had received training in communication:

- Creating a conducive atmosphere for communication;

- Assessing and understanding the patients' mood and TB situation;

- Listening skills;

- Questioning skills;

- Constructive feedback skills;

- Understanding non-verbal communication skills; and

- Expressing respect and empathy to patients. 
In addition, the evaluation results also showed an improvement in TB knowledge of patients with tuberculosis. It indicated that the draft guidelines for communication indeed brought about a significant improvement in the TB program.

\section{Conclusion}

This article focused only on Phase 4 of the study which was the implementation and evaluation of the draft guidelines on communication. The results of training indicated that nurses who undergone training on communication are communicating effectively and the patients understood their message well since the sharing of information are considered.

\section{Competing}

The authors declare that they have no financial or personal relationship(s) which may have inappropriately influenced them in writing this article.

\section{References}

[1] World Health Organisation (WHO). (2003). Treatment of Tuberculosis: Guideline for National programme (3rd Ed.). WHO/CDS/TB/2003:313. Geneva: WHO.

[2] World Health Organisation (WHO). (2006). the Global Plan to Stop TB. 2005-2010. Geneva: WHO.

[3] Ministry of Health and Social Services (MoHSS).(2006). National Guidelines for the Management of Tuberculosis (2nd Ed.). Windhoek: MoHSS.

[4] Ministry of Health and Social Services (MoHSS) (2007/2008).Annual Report: National Tuberculosis and Leprosy Control Program. Windhoek: MoHSS.

[5] Clark. D. (2008).Communication and Leadership. Retrieved August 24, 2009, from http:nwlink.com/ dondark/about/about.html

[6] Ministry of Health and Social Services (MoHSS). (2009/2010).Staff Establishment: Khomas Regional Health Directorate. Windhoek: MoHSS.

[7] Watson E. (2011). System approach workbook for health education and program planning Sudburg: Jones \&Barlet

[8] Rew, L. (2005). Adolescent health.A multidisciplinary approach to theory, research and intervention. University of Texas, Austin, SAGE Publications: Thousand Oak, London, New Delhi.

[9] Pretorius, L. (2008). An educational programme to facilitate critical thinking of student nurses in Namibia.PhD. Thesis University of Namibia Windhoek Namibia. 